\title{
Necessary Conditions for Interpolation by Multivariate Polynomials
}

\author{
Jorge Antezana ${ }^{1} \cdot$ Jordi Marzo² $\cdot$ Joaquim Ortega-Cerdà $^{2}$
}

Received: 20 January 2021 / Revised: 11 April 2021 / Accepted: 24 May 2021 / Published online: 30 August 2021 (c) The Author(s) 2021

\begin{abstract}
Let $\Omega$ be a smooth, bounded, convex domain in $\mathbb{R}^{n}$ and let $\Lambda_{k}$ be a finite subset of $\Omega$. We find necessary geometric conditions for $\Lambda_{k}$ to be interpolating for the space of multivariate polynomials of degree at most $k$. Our results are asymptotic in $k$. The density conditions obtained match precisely the necessary geometric conditions that sampling sets are known to satisfy and are expressed in terms of the equilibrium potential of the convex set. Moreover we prove that in the particular case of the unit ball, for $k$ large enough, there are no bases of orthogonal reproducing kernels in the space of polynomials of degree at most $k$.
\end{abstract}

Keywords Interpolating sequences $\cdot$ Multivariate polynomials $\cdot$ Reproducing kernels

This work is dedicated to the memory of Professor Walter Hayman

Communicated by Tom Carroll.

The first author is supported by the Consejo Nacional de Investigaciones Científicas y Técnicas-Argentina (PIP-152), the Agencia Nacional de Promoción de Ciencia y Tecnología-Argentina (PICT 2015-1505),

Universidad Nacional de La Plata-Argentina (UNLP 11X829), and the Spanish Ministerio de Economía y Competividad (grant MTM2016-75196-P). The second and third authors are supported by the Spanish Ministerio de Economía y Competividad (grant MTM2017-83499-P) and the Generalitat de Catalunya (grant 2017 SGR 358).

Joaquim Ortega-Cerdà

jortega@ub.edu

Jorge Antezana

antezana@mate.unlp.edu.ar

Jordi Marzo

jmarzo@ub.edu

1 Departamento de Matemática, Universidad Nacional de La Plata, and Instituto Argentino de Matemática "Alberto P. Calderón” (IAM-CONICET), Buenos Aires, Argentina

2 Dept. Matemàtica i Informàtica, Universitat de Barcelona and BGSMath, Gran Via 585, 08007 Barcelona, Spain 
Mathematics Subject Classification 42C05 · 41A10 - 41A63

\section{Introduction}

Given a measure $\mu$ in $\mathbb{R}^{n}$ we consider the space $\mathcal{P}_{k}$ of polynomials of total degree at most $k$ in $n$-variables endowed with the natural scalar product in $L^{2}(\mu)$. We assume that the support of $\mu$ is not contained in the zero set of any $p \in \mathcal{P}_{k}, p \neq 0$. In this case, the norm of $L^{2}(\mu)$ is also a norm for the space $\mathcal{P}_{k}$, and the point evaluation at any given point $x \in \mathbb{R}^{n}$ is a bounded linear functional. Hence, the space $\mathcal{P}_{k}$ becomes a reproducing kernel Hilbert space. By the Riesz representation theorem, for any $x \in \mathbb{R}^{n}$, there is a unique function $K_{k}(\mu, x, \cdot) \in \mathcal{P}_{k}$ such that

$$
p(x)=\left\langle p, K_{k}(\mu, x, \cdot)\right\rangle=\int p(y) K_{k}(\mu, x, y) d \mu(y)
$$

for every $p \in \mathcal{P}_{k}$. Given a point $x \in \mathbb{R}^{n}$ the normalized reproducing kernel is denoted by $\kappa_{k, x}$, i.e.

$$
\kappa_{k, x}(\mu, y)=\frac{K_{k}(\mu, x, y)}{\left\|K_{k}(\mu, x, \cdot)\right\|_{L^{2}(\mu)}}=\frac{K_{k}(\mu, x, y)}{\sqrt{K_{k}(\mu, x, x)}}
$$

We will denote by $\beta_{k}(\mu, x)$ the value of the reproducing kernel on the diagonal

$$
\beta_{k}(\mu, x)=K_{k}(\mu, x, x)
$$

The function $1 / \beta_{k}(\mu, x)$ is often called the Christoffel function. For brevity we may sometimes omit the dependence on $\mu$.

Following Shapiro and Shields in [16] we define sampling and interpolating sets:

Definition 1 A sequence $\Lambda=\left\{\Lambda_{k}\right\}$ of finite sets of points in $\mathbb{R}^{n}$ is said to be interpolating for the sequence of spaces $\left(\mathcal{P}_{k}, L^{2}(\mu)\right)_{k \geq 0}$ if the associated family of normalized reproducing kernels at the points $\lambda \in \Lambda_{k}$, i.e. $\kappa_{k, \lambda}$, is a Riesz sequence in the Hilbert space $\mathcal{P}_{k}$, uniformly in $k$. That is, if there is a constant $C>0$ independent of $k$ such that for any linear combination of the normalized reproducing kernels we have:

$$
\frac{1}{C} \sum_{\lambda \in \Lambda_{k}}\left|c_{\lambda}\right|^{2} \leq\left\|\sum_{\lambda \in \Lambda_{k}} c_{\lambda} \kappa_{k, \lambda}\right\|^{2} \leq C \sum_{\lambda \in \Lambda_{k}}\left|c_{\lambda}\right|^{2}
$$

The definition above is usually decoupled into two separate conditions. The left hand side inequality in (1.1) is usually called the Riesz-Fischer property for the reproducing kernels and is equivalent to the condition that the following moment problem is solvable: for arbitrary values $\left\{v_{\lambda}\right\}_{\lambda \in \Lambda_{k}}$ there exists a polynomial $p \in \mathcal{P}_{k}$ such that $p(\lambda) / \sqrt{\beta_{k}(\lambda)}=\left\langle p, \kappa_{k, \lambda}\right\rangle=v_{\lambda}$ for all $\lambda \in \Lambda_{k}$ and 


$$
\|p\|^{2} \leq C \sum_{\lambda \in \Lambda_{k}}\left|v_{\lambda}\right|^{2}=\sum_{\lambda \in \Lambda_{k}} \frac{|p(\lambda)|^{2}}{\beta_{k}(\lambda)} .
$$

This is the reason $\Lambda$ is called an interpolating family.

The right hand side inequality in (1.1) is called the Bessel property for the normalized reproducing kernels $\left\{\kappa_{k, \lambda}\right\}_{\lambda \in \Lambda_{k}}$. The Bessel property is equivalent to having

$$
\sum_{\lambda \in \Lambda_{k}} \frac{|p(\lambda)|^{2}}{\beta_{k}(\lambda)} \leq C\|p\|^{2}
$$

for all $p \in \mathcal{P}_{k}$. That is, if we denote $\mu_{k}:=\sum_{\lambda \in \Lambda_{k}} \delta_{\lambda} / \beta_{k}(\lambda)$, we are requiring that the identity is a uniformly continuous embedding of $\left(\mathcal{P}_{k}, L^{2}(\mu)\right)$ into $\left(\mathcal{P}_{k}, L^{2}\left(\mu_{k}\right)\right)$.

The notion of sampling plays a similar but opposite role.

Definition 2 A sequence $\Lambda=\left\{\Lambda_{k}\right\}$ of finite sets of points in $\mathbb{R}^{n}$ is said to be sampling or Marcinkiewicz-Zygmund for the sequence of spaces $\left(\mathcal{P}_{k}, L^{2}(\mu)\right)_{k \geq 0}$ if each family $\left\{\kappa_{k, \lambda}\right\}_{\lambda \in \Lambda_{k}}$ is a frame for the corresponding Hilbert space $\mathcal{P}_{k}$, and their frame bounds are uniform in $k$. More precisely, if there is a constant $C>0$ independent of $k$ such that for any polynomial $p \in P_{k}$ :

$$
\frac{1}{C} \sum_{\lambda \in \Lambda_{k}}\left|\left\langle p, \kappa_{k, \lambda}\right\rangle\right|^{2} \leq\|p\|^{2} \leq C \sum_{\lambda \in \Lambda_{k}}\left|\left\langle p, \kappa_{k, \lambda}\right\rangle\right|^{2} .
$$

Observe that the left hand side inequality in (1.3) is the Bessel condition mentioned above. If we were considering only a single space of polynomials $\mathcal{P}_{k_{0}}$ then the notion of an interpolating family amounts to the independence of the corresponding reproducing kernels. On the other hand, the notion of a sampling family corresponds to reproducing kernels that span the whole space $\mathcal{P}_{k_{0}}$.

Throughout this paper, $\Omega$ denotes a smooth, bounded, convex domain. We will restrict our attention to two cases:

- In the first case we consider measures $d \mu(x)=\chi_{\Omega}(x) d V(x)$ on $\Omega$, where $d V$ is the Lebesgue measure.

- In the second case, we consider the case of the unit ball $\mathbb{B}=\left\{x \in \mathbb{R}^{n}:|x| \leq 1\right\}$, and measures of the form $d \mu(x)=\left(1-|x|^{2}\right)^{a-1 / 2} \chi_{\mathbb{B}}(x) d V(x)$ where $a \geq 0$.

In these two cases there are good explicit estimates for the size of the reproducing kernel on the diagonal $K_{k}(\mu, x, x)$, and therefore both notions, interpolation and sampling families, become more tangible. In [2] the authors obtained necessary geometric conditions for sampling families in bounded smooth convex sets with weights when the weights satisfy two technical conditions: Bernstein-Markov and moderate growth. These properties are both satisfied for the Lebesgue measure in a convex set. The case of interpolating families in convex sets was not considered, since there were several technical hurdles to applying the same technique.

Our aim in this paper is to fill this gap and obtain necessary geometric conditions for interpolating families in the two settings mentioned above. The geometric conditions that usually appear in this type of problem come in three flavours: 
- A separation condition. This is implied by the Riesz-Fischer condition i.e. the left hand side of (1.1). The requirement that one should be able to interpolate the values one and zero implies that different points $\lambda, \lambda^{\prime} \in \Lambda_{k}$ with $\lambda \neq \lambda^{\prime}$ cannot be too close. The separation conditions in our settings are studied in Sect. 3.1.

- A Carleson-type condition. This is a condition that ensures the continuity of the embedding as in (1.2). A geometric characterization of the Carleson-type condition is given in Theorem 6 for convex domains and the Lebesgue measure, and in Theorem 7 for the ball and the measures $\mu_{a}$.

- A density condition. This is a global condition that usually follows from both the Bessel and the Riesz-Fischer conditions. A necessary density condition for interpolating sequences on convex sets endowed with the Lebesgue measure is provided in our first main result (see next section for precise definitions):

Theorem 1 Let $\Omega$ be a smooth, bounded, convex domain in $\mathbb{R}^{n}$, and let $\Lambda=\left\{\Lambda_{k}\right\}$ be an interpolating sequence. Then for any Euclidean ball $\mathbb{B}(x, r) \subset \Omega$ the following holds:

$$
\limsup _{k \rightarrow \infty} \frac{\#\left(\Lambda_{k} \cap \mathbb{B}(x, r)\right)}{\operatorname{dim} \mathcal{P}_{k}} \leq \mu_{e q}(\mathbb{B}(x, r)) .
$$

Here $\mu_{e q}$ is the normalized equilibrium measure associated to $\Omega$.

In Theorem 8 we consider the case of the ball with the measure $\mu_{a}$. In both cases a matching density result for sampling sequences was proved in [2].

Finally, a natural question is whether or not there exists a family $\left\{\Lambda_{k}\right\}$ that is both sampling and interpolating. To answer this question is very difficult in general (see [14, Sect. 9], [7]). A particular case is when $\left\{\kappa_{k, \lambda}\right\}_{\lambda \in \Lambda_{k}}$ form an orthonormal basis. In the last section we study the existence of orthonormal bases of reproducing kernels in the case of the ball with the measures $\mu_{a}$. More precisely, if the spaces $\mathcal{P}_{k}$ are endowed with the inner product of $L^{2}\left(\mu_{a}\right)$, we prove that for $k$ big enough the space $\mathcal{P}_{k}$ does not admit an orthonormal basis of reproducing kernels:

Theorem 2 Let $\mathbb{B} \subset \mathbb{R}^{n}$ be the unit ball and $n>1$, and fix any $a \geq 0$. There exists a $k_{0}$ big enough such that for any $k \geq k_{0}$ the space $\mathcal{P}_{k}$ does not admit a basis of reproducing kernels that is orthogonal with respect to the inner product induced by the measure $d \mu_{a}=\left(1-|x|^{2}\right)^{a-1 / 2} d V$.

To determine whether or not there exists a family $\left\{\Lambda_{k}\right\}$ that is both sampling and interpolating for $\left(\mathcal{P}_{k}, \mu_{a}\right)$ remains an open problem.

\section{Technical Results}

Before stating and proving our results we will recall the behaviour of the kernel on the diagonal, or equivalently the Christoffel function, we will define an appropriate metric and introduce some tools. 


\subsection{Christoffel Functions and Equilibrium Measures}

To write explicitly the sampling and interpolating conditions we need an estimate for the Christoffel function. In [2] it was observed that in the case of the measure $d \mu(x)=\chi_{\Omega}(x) d V(x)$ it is possible to obtain precise estimates for the size of the reproducing kernel on the diagonal:

Theorem 3 Let $\Omega$ be a smooth bounded convex domain in $\mathbb{R}^{n}$. Then the reproducing kernel for $\left(\mathcal{P}_{k}, \chi_{\Omega} d V\right)$ satisfies

$$
\beta_{k}(x)=K_{k}(x, x) \simeq \min \left(\frac{k^{n}}{\sqrt{d(x, \partial \Omega)}}, k^{n+1}\right) \text { for all } x \in \Omega,
$$

where $d(x, \partial \Omega)$ denotes the Euclidean distance from $x \in \Omega$ to the boundary of $\Omega$.

For the weight $\left(1-|x|^{2}\right)^{a-1 / 2}$ in the ball $\mathbb{B} \subseteq \mathbb{R}^{n}$ the behaviour of the Christoffel function is well known.

Proposition 4 For any $a \geq 0$, let $d \mu_{a}(x)=\left(1-|x|^{2}\right)^{a-1 / 2} \chi_{\mathbb{B}}(x) d V(x)$. Then the reproducing kernel for $\left(\mathcal{P}_{k}, d \mu_{a}\right)$ satisfies

$$
\beta_{k}\left(\mu_{a}, x\right)=K_{k}\left(\mu_{a}, x, x\right) \simeq \min \left(\frac{k^{n}}{d(x, \partial \mathbb{B})^{a}}, k^{n+2 a}\right) \text { for all } x \in \mathbb{B} .
$$

The proof follows from [15, Prop 4.5 and 5.6], the Cauchy-Schwarz inequality and the extremal characterization of the kernel

$$
K_{k}\left(\mu_{a} ; x, x\right)=\sup \left\{|p(x)|^{2}: p \in \mathcal{P}_{k}, \int|p|^{2} d \mu_{a} \leq 1\right\} .
$$

The asymptotic behaviour for the case of the ball was established in [4]. See also [1] for the general case.

To define the equilibrium measure we have to introduce a few concepts from pluripotential theory, see [10]. Let $L \subset \mathbb{R}^{n} \subset \mathbb{C}^{n}$ be a non-pluripolar compact set. Given $\xi \in \mathbb{C}^{n}$, the Siciak extremal function is defined by

$$
G_{L}(\xi)=\sup \left\{\frac{\log ^{+}|p(\xi)|}{\operatorname{deg}(p)}: p \in P\left(\mathbb{C}^{n}\right), \sup _{L}|p| \leq 1\right\},
$$

where $P\left(\mathbb{C}^{n}\right)$ is the space of holomorphic polynomials. The pluricomplex Green's function is the semicontinuous regularization of $G_{L}$ defined for $z \in \mathbb{C}^{n}$ by

$$
G_{L}^{*}(z)=\limsup _{\xi \rightarrow z} G_{L}(\xi)
$$

The pluripotential equilibrium measure for $L$ is the Monge-Ampère Borel (probability) measure

$$
d \mu_{e q}=\left(d d^{c} G_{L}^{*}\right)^{n}
$$


In the general case, when $\Omega$ is a smooth bounded convex domain the equilibrium measure is very well understood, see [3,5]. It behaves roughly as $d \mu_{e q} \simeq 1 / \sqrt{d(x, \partial \Omega)} d V$. In particular, the pluripotential equilibrium measure for the ball $\mathbb{B}$ is given (up to normalization) by $d \mu_{0}(x)=1 / \sqrt{1-|x|^{2}} d V(x)$.

\subsection{An Anisotropic Distance}

The natural distance to formulate the separation condition and the Carleson-type condition is not the Euclidean distance. Consider in the unit ball $\mathbb{B} \subset \mathbb{R}^{n}$ the following distance:

$$
\rho(x, y)=\arccos \left\{\langle x, y\rangle+\sqrt{1-|x|^{2}} \sqrt{1-|y|^{2}}\right\} .
$$

This is the geodesic distance between the points $x^{\prime}, y^{\prime}$ in the sphere $\mathbb{S}^{n}$ defined as $x^{\prime}=\left(x, \sqrt{1-|x|^{2}}\right)$ and $y^{\prime}=\left(y, \sqrt{1-|y|^{2}}\right)$. If we consider anisotropic balls $B(x, \varepsilon)=\{y \in \mathbb{B}: \rho(x, y)<\varepsilon\}$, they are comparable to a box centered at $x$ (a product of intervals) which are of size $\varepsilon$ in the tangent directions and size $\varepsilon^{2}+\varepsilon \sqrt{1-|x|^{2}}$ in the radial direction. If we want to refer to a Euclidean ball of center $x$ and radius $\varepsilon$ we would use the notation $\mathbb{B}(x, \varepsilon)$.

The Euclidean volume of a ball $B(x, \varepsilon)$ is comparable to $\varepsilon^{n} \sqrt{1-|x|^{2}}$ if $\left(1-|x|^{2}\right)>$ $\varepsilon^{2}$ and $\varepsilon^{n+1}$ otherwise.

This distance $\rho$ can be extended to an arbitrary smooth convex domain $\Omega$ by using Euclidean balls contained in $\Omega$ and tangent to the boundary of $\Omega$. This can be done in the following way. Since $\Omega$ is smooth, there is a tubular neighbourhood $U \subset \mathbb{R}^{n}$ of the boundary of $\Omega$ where each point $x \in U$ has a unique closest point $\tilde{x}$ in $\partial \Omega$ and the normal line to $\partial \Omega$ at $\tilde{x}$ passes through $x$. There is a fixed small radius $r>0$ such that any point $x \in U \cap \Omega$ is contained in a ball of radius $r, \mathbb{B}(p, r) \subset \Omega$ and such that it is tangent to $\partial \Omega$ at $\tilde{x}$. We define at $x$ a Riemannian metric which comes from the pullback of the standard metric on $\partial \tilde{\mathbb{B}}(p, r)$ where $\tilde{\mathbb{B}}(p, r)$ is a ball in $\mathbb{R}^{n+1}$ centered at $(p, 0)$ and of radius $r>0$ by the projection of $\mathbb{R}^{n+1}$ onto the first $n$-variables. In this way we have defined a Riemannian metric in the domain $\Omega \cap U$. In the core of $\Omega$, i.e. far from the boundary we use the standard Euclidean metric. We glue the two metrics with a partition of unity.

The resulting metric $\rho$ on $\Omega$ has the relevant property that the balls of radius $\varepsilon$ behave as in the unit ball, that is a ball $B(x, \varepsilon)$ of center $x$ and of radius $\varepsilon$ in this metric is comparable to a box of size $\varepsilon$ in the tangent directions and size $\varepsilon^{2}+\varepsilon \sqrt{d(x, \partial \Omega)}$ in the normal direction.

\subsection{Well Localized Polynomials}

The basic tool that we will use to prove the Carleson-type condition and the separation condition are well localized polynomials. These were studied by Petrushev and $\mathrm{Xu}$ in the unit ball with the measure $d \mu_{a}=\left(1-|x|^{2}\right)^{a-1 / 2} d V$, for $a \geq 0$. We recall their basic properties: 
Theorem A (Petrushev and Xu) Let $d \mu_{a}=\left(1-|x|^{2}\right)^{a-1 / 2} d$ V on $\mathbb{B}$ for $a \geq 0$. For any $k \geq 1$ and any $y \in \mathbb{B} \subset \mathbb{R}^{n}$ there are polynomials $L_{k}^{a}(\cdot, y) \in \mathcal{P}_{2 k}$ that satisfy:

(1) $L_{k}^{a}(x, y)$ as a variable of $x$ is a polynomial of degree $2 k$.

(2) $L_{k}^{a}(x, y)=L_{k}^{a}(y, x)$.

(3) $L_{k}^{a}$ reproduces all the polynomials of degree $k$, i.e.

$$
p(y)=\frac{1}{\mu_{a}(\mathbb{B})} \int_{\mathbb{B}} L_{k}^{a}(x, y) p(x) d \mu_{a}(x), \quad \text { for all } p \in \mathcal{P}_{k} .
$$

(4) For any $\gamma>0$ there is a $c_{\gamma}$ such that

$$
\left|L_{k}^{a}(x, y)\right| \leq c_{\gamma} \frac{\sqrt{\beta_{k}\left(\mu_{a}, x\right) \beta_{k}\left(\mu_{a}, y\right)}}{(1+k \rho(x, y))^{\gamma}} .
$$

(5) The kernels $L_{k}^{a}$ are Lispchitz with respect to the metric $\rho$. More concretely, for all $x \in B(y, 1 / k)$

$$
\left|L_{k}^{a}(w, x)-L_{k}^{a}(w, y)\right| \leq c_{\gamma} \frac{k \rho(x, y) \sqrt{\beta_{k}\left(\mu_{a}, w\right) \beta_{k}\left(\mu_{a}, y\right)}}{(1+k \rho(w, y))^{\gamma}} .
$$

(6) There is $\varepsilon>0$ such that $L_{k}^{a}(x, y) \simeq K_{k}\left(\mu_{a} ; y, y\right)$ for all $x \in B(y, \varepsilon / k)$.

Proof All the properties are proved in [15, Thm 4.2, Prop 4.7 and 4.8] except the behaviour near the diagonal number 6 . Let us start by observing that by the Lipschitz condition (2.5) it is enough to prove that $L_{k}^{a}(x, x) \simeq K_{k}\left(\mu_{a} ; x, x\right)$.

This follows from the definition of $L_{k}^{a}$ which is done as follows. The subspace $V_{k} \subset L^{2}(\mathbb{B})$ are the polynomials of degree $k$ that are orthogonal to lower degree polynomials in $L^{2}(\mathbb{B})$ with respect to the measure $d \mu_{a}$. Consider the kernels $P_{k}(x, y)$ which are the kernels that give the orthogonal projection on $V_{k}$. If $f_{1}, \ldots, f_{r}$ is an orthonormal basis for $V_{k}$ then $P_{k}(x, y)=\sum_{j=1}^{r} f_{j}(x) f_{j}(y)$. The kernel $L_{k}^{a}$ is defined as

$$
L_{k}^{a}(x, y)=\sum_{j=0}^{\infty} \hat{a}\left(\frac{j}{k}\right) P_{j}(x, y) .
$$

We assume that $\hat{a}$ is compactly supported, $\hat{a} \geq 0, \hat{a} \in \mathcal{C}^{\infty}(\mathbb{R})$, supp $\hat{a} \subset[0,2]$, $\hat{a}(t)=1$ on $[0,1]$ and $\hat{a}(t) \leq 1$ on $[1,2]$ as in Fig. 1 . 


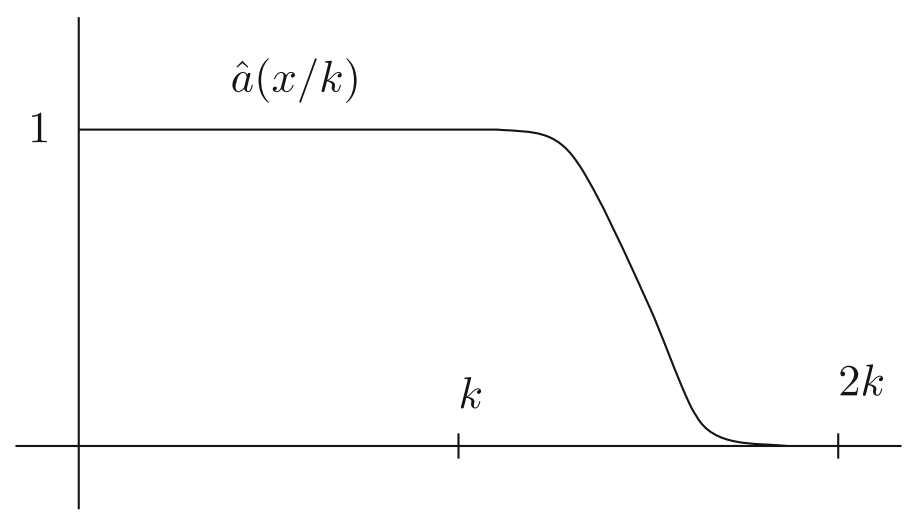

Fig. 1 Graph of $\hat{a}$.

Then, all the terms are positive in the diagonal. Recall that

$$
K_{k}\left(\mu_{a} ; x, y\right)=\sum_{j=0}^{k} P_{j}(x, y)
$$

Hence, we get

$$
\beta_{k}\left(\mu_{a}, x\right)=K_{k}\left(\mu_{a} ; x, x\right) \leq L_{k}^{a}(x, x) \leq K_{2 k}\left(\mu_{a} ; x, x\right)=\beta_{2 k}\left(\mu_{a}, x\right) .
$$

Since $\beta_{k}\left(\mu_{a}, x\right) \simeq \beta_{2 k}\left(\mu_{a}, x\right)$ we obtain the desired estimate.

Petrushev and $\mathrm{Xu}$ also proved the following integral estimate [15, Lem. 4.6]

Lemma B Let $\alpha>0$ and $a \geq 0$. If $\gamma>0$ is big enough we have

$$
\int_{\mathbb{B}} \frac{K_{k}\left(\mu_{a}, y, y\right)^{\alpha}}{(1+k \rho(x, y))^{\gamma}} d \mu_{a}(y) \lesssim \frac{1}{K_{k}\left(\mu_{a}, x, x\right)^{1-\alpha}} .
$$

\section{Main Results}

\subsection{Separation}

In our first result we prove that for $\Lambda=\left\{\Lambda_{k}\right\}$ interpolating there exists $\varepsilon>0$ such that

$$
\inf _{\lambda, \lambda^{\prime} \in \Lambda_{k}, \lambda \neq \lambda^{\prime}} \rho\left(\lambda, \lambda^{\prime}\right) \geq \frac{\varepsilon}{k}
$$


Theorem 5 If $\Omega$ is a smooth, bounded, convex domain and $\Lambda=\left\{\Lambda_{k}\right\}$ is an interpolating sequence for $\left(\mathcal{P}_{k}, L^{2}\left(\chi_{\Omega} d V\right)\right)_{k \geq 0}$ then there is an $\varepsilon>0$ such that the balls $\{B(\lambda, \varepsilon / k)\}_{\lambda \in \Lambda_{k}}$ are pairwise disjoint.

Proof Consider the metric in $\Omega$ defined in Sect. 2.2, and let $r(\Omega)$ be the width of the tubular neighborhood. If $d(\lambda, \partial \Omega) \leq r(\Omega)$, then we take the ball of radius $r(\Omega)$ that contains $\lambda$ and is tangent to $\partial \Omega$ at the closest point to $\lambda$. If $d(\lambda, \partial \Omega)>r(\Omega)$, then we take the ball of radius $r(\Omega)$ centered at $x$. To simplify the notation assume that $r(\Omega)=1$, and in both cases we denote this ball by $\mathbb{B}$. Note that by defining $\mathbb{B}$ in this way, the anisotropic distance in $\mathbb{B}$ is equivalent to the anisotropic distance in $\Omega$. So, we will denote both by $\rho$. Suppose that there is another point $\lambda^{\prime} \in \Lambda_{k} \cap \mathbb{B}$ such that $\rho\left(\lambda, \lambda^{\prime}\right)<1 / k$. Since $\Lambda$ is interpolating we can build a polynomial $p \in \mathcal{P}_{k}$ such that $p\left(\lambda^{\prime}\right)=0, p(\lambda)=1$ and $\|p\|^{2} \lesssim 1 / K_{k}\left(\mu_{1 / 2}, \lambda, \lambda\right)$. In the ball $\mathbb{B}$, the kernel $L_{k}^{1 / 2}$ from Theorem A, for the Lebesgue measure $a=1 / 2$, is reproducing. Therefore

$$
1=\int_{\mathbb{B}}\left(L_{k}^{1 / 2}(\lambda, w)-L_{k}^{1 / 2}\left(\lambda^{\prime}, w\right)\right) p(w) d V(w) .
$$

We can use the estimate

$$
|p(w)| \leq \sqrt{\beta_{k}\left(\mu_{1 / 2}, w\right)}\|p\| \leq \sqrt{\left.\beta_{k}\left(\mu_{1 / 2}, w\right) / \beta_{k}\left(\mu_{1 / 2}, \lambda\right)\right)}
$$

and the inequality (2.5) to obtain

$$
1 \lesssim k \rho\left(\lambda, \lambda^{\prime}\right) \int_{\mathbb{B}} \frac{\beta_{k}\left(\mu_{1 / 2}, w\right) d V(w)}{(1+k \rho(w, \lambda))^{\gamma}},
$$

Taking $\alpha=1$ and $a=1 / 2$ in Lemma B we finally get $1 \lesssim k \rho\left(\lambda, \lambda^{\prime}\right)$ as stated.

Observe that considering the general case $L_{k}^{a}$ in (3.1), one can prove the corresponding result for interpolating sequences for $\mathcal{P}_{k}$ with weight

$$
d \mu_{a}(x)=\left(1-|x|^{2}\right)^{a-1 / 2} d V(x)
$$

in the ball $\mathbb{B}$.

\subsection{Carleson-Type Condition}

Let us deal with condition (1.2).

Definition 3 A sequence of measures $\left\{\mu_{k}\right\}_{k \geq 0}$ are called Carleson measures for $\left(\mathcal{P}_{k}, d \mu\right)$ if there is a constant $C>0$, independent of $k$, such that

$$
\int_{\Omega}|p(x)|^{2} d \mu_{k}(x) \leq C\|p\|_{L^{2}(\mu)}^{2}
$$

for all $p \in \mathcal{P}_{k}$. 


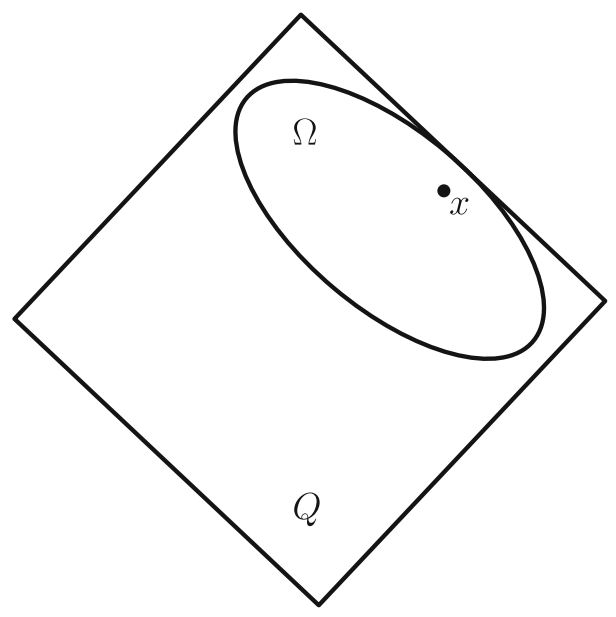

Fig. 2 External tangent cube

In particular if $\Lambda_{k}$ is a sequence of interpolating sets then the sequence of measures $\mu_{k}=\sum_{\lambda \in \Lambda_{k}} \delta_{\lambda} / \beta_{k}(\lambda)$ is Carleson.

The geometric characterization of the Carleson measures when $\Omega$ is a smooth convex domain is in terms of anisotropic balls.

Theorem 6 Let $\Omega$ be a smooth, bounded, convex domain in $\mathbb{R}^{n}$. A sequence of measures $\left\{\mu_{k}\right\}_{k \geq 0}$ is Carleson for $\left(\mathcal{P}_{k}, \chi_{\Omega} d V\right)$ if and only if there is a constant $C>0$, independent of $k$, such that for all points $x \in \Omega$

$$
\mu_{k}(B(x, 1 / k)) \leq C V(B(x, 1 / k)) .
$$

Proof We prove the necessity. For any $x \in \Omega$ there is a cube $Q$ that contains $\Omega$ which is tangent to $\partial \Omega$ at a closest point to $x$ as in Fig. 2 .

This cube has fixed dimensions independent of the point $x \in \Omega$. Define the polynomials $Q_{k}^{x}(y)=L_{k}^{1 / 2}\left(x_{1}, y_{1}\right) \cdots L_{k}^{1 / 2}\left(x_{n}, y_{n}\right)$ of degree at most $k n$. We test the Carleson condition (3.2) against these polynomials that peak at $B(x, 1 / k)$ and we get

$$
\int_{B(x, 1 / k)}\left|Q_{k}^{x}\right|^{2} d \mu_{k} \leq \int_{\Omega}\left|Q_{k}^{x}\right|^{2} d \mu_{k} \leq C\left\|Q_{k}^{x}\right\|_{L^{2}(Q)}^{2} .
$$

By property (6) in Theorem A the left hand side is bounded below by

$$
C \mu_{k}(B(x, 1 / k)) \prod_{i=1}^{n} K_{k}\left(\mu_{1 / 2} ; x_{i}, x_{i}\right)^{2}
$$

For the right hand side we split the product of integrals and use that

$$
\int L_{k}^{1 / 2}\left(x_{i}, y_{i}\right)^{2} d \mu_{1 / 2}\left(y_{i}\right) \simeq K_{k}\left(\mu_{1 / 2} ; x_{i}, x_{i}\right) \text {. }
$$


Finally the estimate (2.2) applied to these one dimensional kernels and the fact that

$$
V(B(x, 1 / k)) \simeq \frac{1}{k^{n}}\left(\sqrt{1-|x|^{2}}+\frac{1}{k}\right)
$$

gives the necessary condition.

For the sufficiency we use the reproducing property of $L_{k}^{1 / 2}(z, y)$. For any point $x \in \Omega$ there is a Euclidean ball $\mathbb{B}_{x}$ contained in $\Omega$ such that $x \in \mathbb{B}_{x}$ and is tangent to $\partial \Omega$ at the closest point to $x$. Moreover, since $\Omega$ is a smooth bounded convex domain we can assume that the radius of $\mathbb{B}_{x}$ has a lower bound independent of $x$. In this ball we can reconstruct the square of any polynomial $p \in \mathcal{P}_{k}$ using the kernel $L_{2 k}^{1 / 2}$ relative to the ball $\mathbb{B}_{x}$. That is

$$
\int_{\Omega}|p(x)|^{2} d \mu_{k}(x) \leq \int_{\Omega}\left|\int_{\mathbb{B}_{x}} L_{2 k}^{1 / 2}(x, y) p^{2}(y) d V(y)\right| d \mu_{k}(x) .
$$

We use the estimate (2.4) and we get

$$
\int_{\Omega}|p(x)|^{2} d \mu_{k}(x) \lesssim \int_{\Omega} \int_{\mathbb{B}_{x}} \frac{\sqrt{\beta_{2 k}(x) \beta_{2 k}(y)}}{(1+2 k \rho(x, y))^{\gamma}}|p(y)|^{2} d V(y) d \mu_{k}(x) .
$$

We break the integral in two regions, when $\rho(x, y)<1$ and otherwise. When $\gamma$ is big enough we obtain:

$$
\begin{aligned}
\int_{\Omega}|p(x)|^{2} d \mu_{k}(x) \lesssim & \int_{\Omega} \int_{\mathbb{B}_{x} \cap \rho(x, y)>1}|p(y)|^{2} d V(y) d \mu_{k}(x)+ \\
& \int_{\Omega} \int_{\mathbb{B}_{x} \cap \rho(x, y)<1} \frac{\sqrt{\beta_{2 k}(x) \beta_{2 k}(y)}}{(1+2 k \rho(x, y))^{\gamma}}|p(y)|^{2} d V(y) d \mu_{k}(x) .
\end{aligned}
$$

The first integral on the right hand side is bounded by $\int_{\Omega}|p(y)|^{2} d V(y)$ since $\mu_{k}(\Omega)$ is bounded by hypothesis (it is possible to cover $\Omega$ by balls $\left\{B\left(x_{n}, 1 / k\right)\right\}$ with controlled overlap).

In the second integral, observe that if $w \in B(x, 1 / k)$ then $\rho(w, x) \leq 1 / k$ and therefore

$$
\frac{\sqrt{\beta_{2 k}(x) \beta_{2 k}(y)}}{(1+2 k \rho(x, y))^{\gamma}} \lesssim \frac{1}{V(B(x, 1 / k))} \int_{B(x, 1 / k)} \frac{\sqrt{\beta_{2 k}(w) \beta_{2 k}(y)}}{(1+2 k \rho(w, y))^{\gamma}} d V(w) .
$$

We plug this inequality into the second integral and we can bound it by

$$
\int_{\Omega}|p(y)|^{2} \int_{\rho(w, y)<2} \frac{\sqrt{\beta_{2 k}(w) \beta_{2 k}(y)}}{(1+2 k \rho(w, y))^{\gamma}} \frac{\mu_{k}(B(w, 1 / k))}{V(B(w, 1 / k))} d V(w) d V(y) .
$$

We use the hypothesis (3.3) and Lemma B with $\alpha=1 / 2$ to bound it finally by $\int_{\Omega}|p(y)|^{2} d V(y)$. 
The weighted case in the unit ball is simpler.

Theorem 7 Let $d \mu_{a}(x)=\left(1-|x|^{2}\right)^{a-1 / 2} d V(x)$ for $a \geq 0$ be the weight in the unit ball $\mathbb{B} \subset \mathbb{R}^{n}$. A sequence of measures $\left\{\mu_{k}\right\}_{k \geq 0}$ is Carleson for $\left(\mathcal{P}_{k}, \mu_{a}\right)_{k \geq 0}$ if and only if there is a constant $C$, independent of $k$, such that for all points $x \in \mathbb{B}$

$$
\mu_{k}(B(x, 1 / k)) \leq C \mu_{a}(B(x, 1 / k))
$$

Proof Suppose that $\left\{\mu_{k}\right\}_{k \geq 0}$ is a Carleson sequence of measures. Then for any $x \in \mathbb{B}$

$$
\int_{B(x, 1 / k)}\left|L_{k}^{a}(x, w)\right|^{2} d \mu_{2 k}(w) \leq C\left\|L_{k}^{a}(x, \cdot)\right\|_{L^{2}\left(\mu_{a}\right)}^{2} .
$$

By the definition of the polynomials $L_{k}^{a}$ we get

$$
\begin{aligned}
\left\|L_{k}^{a}(x, \cdot)\right\|_{L^{2}\left(\mu_{a}\right)}^{2} & =\sum_{j_{1}, j_{2}=0}^{\infty} \hat{a}\left(\frac{j_{1}}{k}\right) \hat{a}\left(\frac{j_{2}}{k}\right) \int_{\mathbb{B}} P_{j_{1}}(x, y) P_{j_{2}}(x, y) d \mu_{a}(y) \\
& =\sum_{j=0}^{\infty}\left|\hat{a}\left(\frac{j}{k}\right)\right|^{2} \int_{\mathbb{B}}\left|P_{j}(x, y)\right|^{2} d \mu_{a}(y) \quad \text { by orthogonality } \\
& =\sum_{j=0}^{\infty}\left|\hat{a}\left(\frac{j}{k}\right)\right|^{2} P_{j}(x, x) \text { by the projection property. }
\end{aligned}
$$

Since $\hat{a}(t) \leq 1, \hat{a}(t)=1$ on $[0,1]$, and the support of $\hat{a}$ is contained in $[0,2]$ we obtain the following estimates

$$
K_{k}\left(\mu_{a}, x, x\right) \leq\left\|L_{k}^{a}(x, \cdot)\right\|_{L^{2}\left(\mu_{a}\right)}^{2} \leq K_{2 k}\left(\mu_{a}, x, x\right),
$$

From these estimates and property (6) in Theorem A we get inequality (3.4). The necessity follows exactly as in the unweighted case with the obvious changes.

\subsection{Density Condition}

In [2, Thm. 4] a necessary density condition for sampling sequences for polynomials in convex domains was obtained. It states the following:

Theorem $\mathbf{C}\left(\right.$ Berman and Ortega-Cerdà) Let $\Omega$ be a smooth convex domain in $\mathbb{R}^{n}$, and let $\Lambda$ be a sampling sequence. Then for any $\mathbb{B}(x, r) \subset \Omega$ the following holds:

$$
\liminf _{k \rightarrow \infty} \frac{\#\left(\Lambda_{k} \cap \mathbb{B}(x, r)\right)}{\operatorname{dim} \mathcal{P}_{k}} \geq \mu_{e q}(\mathbb{B}(x, r)) .
$$

Here $\mu_{e q}$ is the normalized equilibrium measure associated to $\Omega$. 
Let us see how, with a similar technique, a corresponding density condition can be obtained as well in the case of interpolating sequences.

Proof (Proof of Theorem 1) Let $F_{k} \subset \mathcal{P}_{k}$ be the subspace spanned by

$$
\kappa_{\lambda}(x)=\frac{K_{k}(\lambda, x)}{\sqrt{\beta_{k}(\lambda)}} \quad \text { for all } \lambda \in \Lambda_{k} .
$$

Denote by $g_{\lambda}$ the dual (biorthogonal) basis to $\kappa_{\lambda}$ in $F_{k}$. We have clearly that

- We can span any function in $F_{k}$ in terms of $\kappa_{\lambda}$, thus:

$$
\sum_{\lambda \in \Lambda_{k}} \kappa_{\lambda}(x) g_{\lambda}(x)=\mathcal{K}_{k}(x, x)
$$

where $\mathcal{K}_{k}(x, y)$ is the reproducing kernel of the subspace $F_{k}$.

- The norm of $g_{\lambda}$ is uniformly bounded since $\kappa_{\lambda}$ was a uniform Riesz sequence.

- $g_{\lambda}(\lambda)=\sqrt{\beta_{k}(\lambda)}$. This is due to the biorthogonality and the reproducing property.

We are going to prove that the measure $\sigma_{k}=\left(1 / \operatorname{dim} \mathcal{P}_{k}\right) \sum_{\lambda \in \Lambda_{k}} \delta_{\lambda}$, and the measure $d v_{k}=\left(1 / \operatorname{dim} \mathcal{P}_{k}\right) \mathcal{K}_{k}(x, x) d V(x)$ are very close to each other. These are two positive measures that are not probability measures but they have the same mass (equal to $\left.\# \Lambda_{k} / \operatorname{dim} \mathcal{P}_{k} \leq 1\right)$. Therefore, there is a way to quantify the closeness through the Vaserstein 1-distance. For an introduction to Vaserstein distance see for instance [17]. We want to prove that $W\left(\sigma_{k}, v_{k}\right) \rightarrow 0$ because the Vaserstein distance metrizes the weak-* topology.

In this case, it is known that $\mathcal{K}_{k}(x, x) \leq K_{k}(x, x)$ and $\left(1 / \operatorname{dim} \mathcal{P}_{k}\right) \beta_{k}(x) \rightarrow \mu_{e q}$ in the weak-* topology, where $\mu_{e q}$ is the normalized equilibrium measure associated to $\Omega$ (see for instance [1]). Therefore, $\lim \sup _{k} \sigma_{k} \leq \mu_{e q}$.

In order to prove that $W\left(\sigma_{k}, v_{k}\right) \rightarrow 0$ we use a signed transport plan as in [12]:

$$
\rho_{k}(x, y)=\frac{1}{\operatorname{dim} \mathcal{P}_{k}} \sum_{\lambda \in \Lambda_{k}} \delta_{\lambda}(y) \times g_{\lambda}(x) \kappa_{\lambda}(x) d V(x)
$$

It has the right marginals, $\sigma_{k}$ and $v_{k}$ and we can estimate the integral

$$
W\left(\sigma_{k}, v_{k}\right) \leq \iint_{\Omega \times \Omega}|x-y| d\left|\rho_{k}\right|=O(1 / \sqrt{k}) .
$$

The only point that merits a clarification is that we need an inequality:

$$
\begin{aligned}
\frac{1}{\operatorname{dim} \mathcal{P}_{k}} \sum_{\lambda \in \Lambda_{k}} \int_{\Omega}|\lambda-x|^{2} & \frac{\left|K_{k}(\lambda, x)\right|^{2}}{K_{k}(x, x)} d V(x) \leq \\
& \frac{1}{\operatorname{dim} \mathcal{P}_{k}} \iint_{\Omega \times \Omega}|y-x|^{2}\left|K_{k}(y, x)\right|^{2} d V(x) d V(y) .
\end{aligned}
$$


This is problematic. We know that $\Lambda_{k}$ is an interpolating sequence for the polynomials of degree $k$. Thus the normalized reproducing kernels at $\lambda \in \Lambda_{k}$ form a Bessel sequence for $\mathcal{P}_{k}$ but the inequality that we need is applied to $K_{k}(x, y)\left(y_{i}-x_{i}\right)$ for all $i=$ $1, \ldots, n$, that is to a polynomial of degree $k+1$. We are going to show that if $\Lambda_{k}$ is an interpolating sequence for the polynomials of degree $k$ it is also a Carleson sequence for the polynomials of degree $k+1$.

Observe that since it is interpolating then it is uniformly separated, i.e. $B(\lambda, \varepsilon / k)$ are disjoint. That means that in particular

$$
\mu_{k}(B(z, 1 /(k+1)) \lesssim V(B(z, 1 /(k+1))
$$

Thus $\mu_{k}$ is a Carleson measure for $\mathcal{P}_{k+1}$.

Finally in [2, Thm. 17] it was proved that

$$
\frac{1}{\operatorname{dim} \mathcal{P}_{k}} \iint_{\Omega \times \Omega}|y-x|^{2}\left|K_{k}(y, x)\right|^{2} d V(x) d V(y)=O(1 / k) .
$$

From the behaviour on the diagonal of the kernel (2.2) its easy to check that the kernel is both Bernstein-Markov (sub-exponential) and has moderate growth, see definitions in [2]. From the characterization of sampling sequences proved in [2, Thm. 1] and with the obvious changes in the proof of the previous theorem we deduce the following:

Theorem 8 Consider the space of polynomials $\mathcal{P}_{k}$ restricted to the ball $\mathbb{B} \subset \mathbb{R}^{n}$ with the measure $d \mu_{a}(x)=\left(1-|x|^{2}\right)^{a-1 / 2} d V$. Let $\Lambda=\left\{\Lambda_{k}\right\}$ be a sequence of sets of points in $\mathbb{B}$.

- If $\Lambda$ is a sampling sequence then, for each $x \in \mathbb{B}$ and $r>0$

$$
\liminf _{k \rightarrow \infty} \frac{\#\left(\Lambda_{k} \cap \mathbb{B}(x, r)\right)}{\operatorname{dim} \mathcal{P}_{k}} \geq \mu_{e q}(\mathbb{B}(x, r)) .
$$

- If $\Lambda$ is interpolating sequence then, for each $x \in \mathbb{B}$ and $r>0$

$$
\limsup _{k \rightarrow \infty} \frac{\#\left(\Lambda_{k} \cap \mathbb{B}(x, r)\right)}{\operatorname{dim} \mathcal{P}_{k}} \leq \mu_{e q}(\mathbb{B}(x, r)) .
$$

Remark In the statements of Theorems $1, C$ and 8 we could have replaced $\mathbb{B}(x, r)$ by any open set, in particular they could have been formulated with balls $B(x, r)$ in the anisotropic metric.

One can construct interpolation or sampling sequences with density arbitrarily close to the critical density with sequences of points $\left\{\Lambda_{k}\right\}$ such that the corresponding Lagrange interpolating polynomials are uniformly bounded. In particular the above inequalities are sharp. For a similar construction on the sphere see [13]. 


\subsection{Orthonormal Basis of Reproducing Kernels}

Sampling and interpolation are somehow dual concepts. Sequences which are both sampling and interpolating (i.e. complete interpolating sequences) are optimal in some sense because they are at the same time minimal sampling sequences and maximal interpolating sequences. In the setting of Theorem 8 , such sequences satisfy

$$
\lim _{k \rightarrow \infty} \frac{\#\left(\Lambda_{k} \cap \mathbb{B}(x, r)\right)}{\operatorname{dim} \mathcal{P}_{k}}=\mu_{e q}(\mathbb{B}(x, r)) .
$$

In general domains, to prove or disprove the existence of such sequences is a difficult problem [14].

If $\Lambda=\left\{\Lambda_{k}\right\}$ is a complete interpolating sequence the corresponding reproducing kernels $\left\{\kappa_{k, \lambda}\right\}$ form a Riesz basis in the space of polynomials (uniformly in the degree). An obvious example of complete interpolating sequences would be sequences providing an orthonormal basis of reproducing kernels. In dimension 1, with the weight $\left(1-x^{2}\right)^{a-1 / 2}$, a basis of Gegenbauer polynomials $\left\{G_{j}^{(a)}\right\}_{j=0, \ldots, k}$ is orthogonal and the reproducing kernel in $\mathcal{P}_{k}$ evaluated at the zeros of the polynomial $G_{k+1}^{(a)}$ gives an orthogonal sequence. In our last result we prove that in higher dimensions there is no orthogonal basis of $\mathcal{P}_{k}$ consisting of reproducing kernels with the measure $d \mu_{a}(x)=\left(1-|x|^{2}\right)^{a-1 / 2} d V(x)$.

Our first goal is to show that sampling sequences are dense enough, Proposition 10. Recall that in $\mathbb{B}(0, r)$, for $r<1$, the Euclidean metric and the metric $\rho$ are equivalent. In our first result we prove that the right hand side of (1.3) and the separation condition imply that there are points of the sequence in any ball $\mathbb{B}(0, r)$ that has a sufficiently large radius.

Proposition 9 Let $d \mu_{a}(x)=\left(1-|x|^{2}\right)^{a-1 / 2} d V(x)$ for $a \geq 0$ be the weight in the unit ball $\mathbb{B} \subset \mathbb{R}^{n}$. Let $\Lambda_{k} \subset \mathbb{B}$ be a finite subset and $C, \varepsilon>0$ be constants such that

$$
\int_{\mathbb{B}}|P(x)|^{2} d \mu_{a}(x) \leq C \sum_{\lambda \in \Lambda_{k}} \frac{|P(\lambda)|^{2}}{K_{k}\left(\mu_{a} ; \lambda, \lambda\right)}
$$

for all $P \in \mathcal{P}_{k}$ and

$$
\inf _{\substack{\lambda, \lambda^{\prime} \in \Lambda_{k} \\ \lambda \neq \lambda^{\prime}}} \rho\left(\lambda, \lambda^{\prime}\right) \geq \frac{\varepsilon}{k}
$$

Given $\left|x_{0}\right|=C_{0}<1 / 4$, there exists $A>0$ depending only on $C, \varepsilon, n$ and $a$ such that

$$
\Lambda_{k} \cap \mathbb{B}\left(x_{0}, A / k\right) \neq \emptyset .
$$


Proof By the construction of the function $L_{\ell}^{a}(x, y)$, as we have already mentioned, for any $\ell \geq 0$

$$
K_{\ell}\left(\mu_{a} ; x, x\right) \leq \int_{\mathbb{B}} L_{\ell}^{a}(x, y)^{2} d \mu_{a}(y) \leq K_{2 \ell}\left(\mu_{a} ; x, x\right) .
$$

Let $P(x)=L_{[k / 2]}^{a}\left(x, x_{0}\right) \in \mathcal{P}_{k}$. Suppose that for some $M>\varepsilon$ and $k \geq 1$

$$
\Lambda_{k} \cap \mathbb{B}\left(x_{0}, M / k\right)=\emptyset .
$$

From the property above, the hypothesis and Proposition 4 we get

$$
k^{n} \simeq K_{[k / 2]}\left(\mu_{a} ; x_{0}, x_{0}\right) \leq \int_{\mathbb{B}} P(y)^{2} d \mu_{a}(y) \lesssim \sum_{\left|\lambda-x_{0}\right|>M / k} \frac{|P(\lambda)|^{2}}{K_{k}\left(\mu_{a} ; \lambda, \lambda\right)}
$$

From [6, Lem. 11.3.6.], given $x \in \mathbb{B}$ and $0<r<\pi$

$$
\mu_{a}(B(x, r)) \simeq r^{n}\left(\sqrt{1-|x|^{2}}+r\right)^{2 a},
$$

and therefore

$$
\mu_{a}(B(x, r)) \simeq \begin{cases}r^{n+2 a} & \text { if } 1-|x|^{2}<r^{2} \\ r^{n}\left(1-|x|^{2}\right)^{a} & \text { otherwise }\end{cases}
$$

and

$$
\mu_{a}(B(x, r)) \gtrsim \begin{cases}r^{n+2 a} & \text { if }|x|>\frac{1}{2} \\ r^{n} & \text { otherwise. }\end{cases}
$$

From (4) in Theorem A, the separation of the sequence, and the estimate (3.10) we get

$$
\begin{aligned}
0 & <c \leq \sum_{\left|\lambda-x_{0}\right|>M / k} \frac{1}{\left(1+[k / 2] \rho\left(x_{0}, \lambda\right)\right)^{2 \gamma}} \\
& =\sum_{\left|\lambda-x_{0}\right|>M / k} \frac{1}{\mu_{a}(B(\lambda, \varepsilon / 2 k))} \int_{B(\lambda, \varepsilon / 2 k)} \frac{d \mu_{a}(x)}{\left(1+[k / 2] \rho\left(x_{0}, \lambda\right)\right)^{2 \gamma}} \\
& \lesssim\left[\sum_{\frac{M}{k}<\left|\lambda-x_{0}\right|<\frac{1}{2}}+\sum_{\frac{1}{2}<\left|\lambda-x_{0}\right|}\right] \frac{1}{\mu_{a}(B(\lambda, \varepsilon / 2 k))} \int_{B(\lambda, \varepsilon / 2 k)} \frac{d \mu_{a}(x)}{\left(1+2 k \rho\left(x_{0}, x\right)\right)^{2 \gamma}} \\
& \lesssim\left(\frac{k}{\varepsilon}\right)^{n} \int_{M / k}^{3 / 4} \frac{r^{n-1}}{(k r)^{2 \gamma}} d r+\frac{k^{2 a+n-2 \gamma}}{\varepsilon^{2 a+n}} \mu_{a}\left(B(0,1 / 2)^{c}\right)
\end{aligned}
$$


Now, for $\gamma=n+a$ we get

$$
0<c \leq \frac{1}{k^{n+2 a}}\left[-\frac{1}{r^{n+2 a}}\right]_{r=M / k}^{3 / 4}+\frac{1}{k^{n}},
$$

and then a uniform (i.e. independent of $k$ ) upper bound for $M$. Taking $A=$ $A(C, \varepsilon, n, a)>M$ we get the result.

Proposition 10 Let $\Lambda=\left\{\Lambda_{k}\right\}$ be a separated sampling sequence for $\mathbb{B} \subset \mathbb{R}^{n}$. Then there exist $M_{0}>0$ such that for any $M>M_{0}$ and all $k \geq 4 M$

$$
\#\left(\Lambda_{k} \cap \mathbb{B}(0, M / k)\right) \simeq M^{n}
$$

Proof Let $\varepsilon>0$ be the constant from the separation, i.e.

$$
\inf _{\substack{\lambda, \lambda^{\prime} \in \Lambda_{k} \\ \lambda \neq \lambda^{\prime}}} \rho\left(\lambda, \lambda^{\prime}\right) \geq \frac{\varepsilon}{k} .
$$

Assume that $M / k \leq 1 / 2$. For $\lambda \in \Lambda_{k} \cap \mathbb{B}(0, M / k)$ we have $V(\mathbb{B}(\lambda, \varepsilon / k)) \simeq(\varepsilon / k)^{n}$ and therefore

$$
\#\left(\Lambda_{k} \cap \mathbb{B}(0, M / k)\right)\left(\frac{\varepsilon}{k}\right)^{n} \lesssim\left(\frac{M}{k}\right)^{n}
$$

For the other inequality, take the constant $A$ (assume $A>\varepsilon$ ) given in Proposition 9 depending on the sampling and the separation constants of $\Lambda$ and $n$. For $M>A$ and $k>0$ such that $\mathbb{B}(0, M / k) \subset \mathbb{B}(0,1 / 4)$ one can find $N$ disjoint balls $\mathbb{B}\left(x_{j}, A / k\right)$ for $j=1, \ldots, N$ contained in $\mathbb{B}(0, M / k)$ and such that

$$
N V(\mathbb{B}(0, A / k))>\frac{1}{2} V(\mathbb{B}(0, M / k)) .
$$

Observe that by Proposition 9 each ball $\mathbb{B}\left(x_{j}, A / k\right)$ contains at least one point from $\Lambda_{k}$ and therefore

$$
\#\left(\Lambda_{k} \cap \mathbb{B}(0, M / k)\right) \geq N \gtrsim\left(\frac{M}{A}\right)^{n} .
$$

We will use the following result from [9].

Theorem $\mathbf{D}$ Let $\mathbb{B} \subset \mathbb{R}^{n}, n>1$, be the unit ball. There do not exist infinite subsets $\Lambda \subset \mathbb{R}^{n}$ such that the exponentials $e^{i\langle x, \lambda\rangle}, \lambda \in \Lambda$, are pairwise orthogonal in $L^{2}(\mathbb{B})$. Or, equivalently, there do not exist infinite subsets $\Lambda \subset \mathbb{R}^{n}$ such that $\left|\lambda-\lambda^{\prime}\right|$ is a zero of $J_{n / 2}$, the Bessel function of order $n / 2$, for all distinct $\lambda, \lambda^{\prime} \in \Lambda$. 
Following ideas from [8] we can prove now our main result about orthogonal bases, Theorem 2. A similar argument can be used on the sphere to study tight spherical designs.

Proof of Theorem 2 The following result can be deduced from [11, Thm. 1.7]: Given $u, v \in \mathbb{R}^{n}$, consider two sequences $\left\{u_{k}\right\}_{k}$ and $\left\{v_{k}\right\}_{k}$ in $\mathbb{R}^{n}$ that converge to $u$ and $v$ respectively, and such that $u_{k} / k, v_{k} / k \in \mathbb{B}$ for every $k \geq 1$. Then

$$
\lim _{k \rightarrow \infty} \frac{K_{k}\left(\mu_{a}, \frac{u_{k}}{k}, \frac{v_{k}}{k}\right)}{K_{k}\left(\mu_{a}, 0,0\right)}=\frac{J_{n / 2}^{*}(|u-v|)}{J_{n / 2}^{*}(0)} .
$$

Let $\Lambda_{k}$ be such that $\left\{\kappa_{\lambda}\right\}_{\lambda \in \Lambda_{k}}$ is an orthonormal basis of $\mathcal{P}_{k}$ with respect to the measure $d \mu_{a}=\left(1-|x|^{2}\right)^{a-1 / 2} d V$. Then

$$
K_{k}\left(\mu_{a}, \lambda_{k}, \lambda_{k}^{\prime}\right)=0
$$

for $\lambda_{k} \neq \lambda_{k}^{\prime} \in \Lambda_{k}$.

We know that $\Lambda_{k}$ is uniformly separated for some $\varepsilon>0$

$$
\rho\left(\lambda_{k}, \lambda_{k}^{\prime}\right) \geq \frac{\varepsilon}{k}
$$

Then the sets $X_{k}=k\left(\Lambda_{k} \cap \mathbb{B}(0,1 / 2)\right) \subset \mathbb{R}^{n}$ are uniformly separated

$$
\left|\lambda-\lambda^{\prime}\right| \gtrsim \varepsilon, \quad \lambda \neq \lambda^{\prime} \in X_{k}
$$

and $X_{k}$ converges weakly to some uniformly separated set $X \subset \mathbb{R}^{n}$. The limit is not empty because by Proposition 10 for any $M>0$,

$$
\#\left(\Lambda_{k} \cap \mathbb{B}(0, M / k)\right) \simeq M^{d} .
$$

Observe that this last result would be a direct consequence of the necessary density condition for complete interpolating sets if we could take balls of radius $r / n$ for a fixed $r>0$ in the condition. Finally, we obtain an infinite set $X$ such that for $u \neq v \in X$

$$
J_{n / 2}^{*}(|u-v|)=0
$$

in contradiction with Theorem D.

Remark Note that the fact that the interpolating sequence $\left\{\Lambda_{k}\right\}$ is complete was used only to guarantee that $\#\left(\Lambda_{k} \cap \mathbb{B}(0, M / k)\right) \simeq M^{d}$. So, the above result could be extended to sequences $\left\{\Lambda_{k}\right\}$ such that $\left\{\kappa_{k, \lambda}\right\}_{\lambda \in \Lambda_{k}}$ is orthonormal (but not necessarily a basis for $\left.\mathcal{P}_{k}\right)$ if $\Lambda_{k} \cap \mathbb{B}(0, M / k)$ contains enough points.

Acknowledgements We would like to thank the referee for a careful review of the paper.

Funding Open Access funding provided thanks to the CRUE-CSIC agreement with Springer Nature. 
Open Access This article is licensed under a Creative Commons Attribution 4.0 International License, which permits use, sharing, adaptation, distribution and reproduction in any medium or format, as long as you give appropriate credit to the original author(s) and the source, provide a link to the Creative Commons licence, and indicate if changes were made. The images or other third party material in this article are included in the article's Creative Commons licence, unless indicated otherwise in a credit line to the material. If material is not included in the article's Creative Commons licence and your intended use is not permitted by statutory regulation or exceeds the permitted use, you will need to obtain permission directly from the copyright holder. To view a copy of this licence, visit http://creativecommons.org/licenses/by/4.0/.

\section{References}

1. Berman, R., Boucksom, S., Nyström, D.W.: Fekete points and convergence towards equilibrium measures on complex manifolds. Acta Math. 207(207), 1-27 (2011)

2. Berman, R., Ortega-Cerdà, J.: Sampling of real multivariate polynomials and pluripotential theory. Am. J. Math. 140(3), 789-820 (2018)

3. Bedford, E., Taylor, A.: The complex equilibrium measure of a symmetric convex set in $\mathbb{R}^{n}$. Trans. Am. Math. Soc. 294, 705-717 (1986)

4. Bos, L.: Asymptotics for the Christoffel function for Jacobi like weights on a ball in $\mathbb{R}^{m}$. N. Z. J. Math. 23(2), 99-109 (1994)

5. Burns, D., Levenberg, N., Ma’u, S., Révész, SZ.: Monge-Ampère measures for convex bodies and Bernstein-Markov type inequalities. Trans. Am. Math. Soc. 362(12), 6325-6340 (2010)

6. Dai, F., Xu, Y.: Approximation theory and harmonic analysis on spheres and balls. Springer Monographs in Mathematics, Springer, New York (2013)

7. Debernardi, A., Lev, N.: Riesz bases of exponentials for convex polytopes with symmetric faces. J. Eur. Math. Soc. JEMS (in press)

8. Fuglede, B.: Commuting self-adjoint partial differential operators and a group theoretic problem. J. Funct. Math. 16, 101-121 (1974)

9. Fuglede, B.: Orthogonal exponentials on the ball. Expo. Math. 19, 267-272 (2001)

10. Klimek, M.: Pluripotential Theory. Oxford University Press, New York (1991)

11. Kroó, A., Lubinsky, D.S.: Christoffel functions and universality in the bulk for multivariate orthogonal polynomials. Can. J. Math. 65(3), 600-620 (2013)

12. Lev, N., Ortega-Cerdà, J.: Equidistribution estimates for Fekete points on complex manifolds. J. Eur. Math. Soc. (JEMS) 18(2), 425-464 (2016)

13. Marzo, J., Ortega-Cerdà, J.: Equidistribution of the Fekete points on the sphere. Constr. Approx. 32, 513-521 (2010)

14. Olevskii, A., Ulanovskii, A.: Functions with disconnected spectrum. Sampling, interpolation, translates, University Lecture Series, 65. American Mathematical Society, Providence, RI (2016)

15. Petrushev, P., Xu, Y.: Localized polynomial frames on the ball. Constr. Approx. 27, 121-148 (2008)

16. Shapiro, H.S., Shields, A.L.: On some interpolation problems for analytic functions. Am. J. Math. 83, 513-532 (1961)

17. Villani, C.: Optimal transport. Grundlehren der Mathematischen Wissenschaften, vol. 338. SpringerVerlag, Berlin (2009)

18. Xu, Y.: Summability of Fourier orthogonal series for Jacobi weight on a ball in $\mathbb{R}^{d}$. Trans. Am. Math. Soc. 351, 2439-2458 (1999)

Publisher's Note Springer Nature remains neutral with regard to jurisdictional claims in published maps and institutional affiliations. 